\section{SKIN MICROCIRCULATION IN HEALTHY NEWBORNS - ASSESSMENTS OF MORPHOLOGY, PERFUSION AND OXYGENATION}

S. Fredly ${ }^{1,2}$, E. Häggblad ${ }^{1,3}$, K. Kvernebo ${ }^{2,4}$, D. Fugelseth ${ }^{1,2}$

${ }^{1}$ Dept. of Neonatal Intensive Care Unit, Oslo University Hospital, Ullevål, ${ }^{2}$ Faculty of Medicine, University of Oslo, Oslo, Norway, ${ }^{3}$ Dept. of Biomedical Engineering, Linköping University, Linköping, Sweden, ${ }^{4}$ Dept. of Cardiothoracic Surgery, Oslo University Hospital, Ullevål, Oslo, Norway

Background and aims: Measurements of central hemodynamics, blood gases and urinary output are commonly used to monitor patients, but values within reference range do not guarantee adequate cellular nutrition and oxygenation. We have examined skin microvascular perfusion, morphology and oxygenation in healthy newborns.

Material and methods: Twenty-five healthy newborns (gestational age $>37$ weeks) were enrolled within 24 hours after delivery. Skin microvascular assessments were performed day one, two and three, on the chest and the hand, using three different techniques: Computer-assisted video microscopy, CAVM (morphology); Laser Doppler Perfusion Monitoring, LDPM (perfusion); Diffuse Reflectance Spectroscopy, DRS (oxygen saturation of capillary erythrocytes).

Results: (Mean with SD) CAVM: Microvascular density (number of microvessels crossing a grid of lines/mm line): Chest: 11.3 (1.5), 11.0 (1.7), 10.7 (1.6). Hand: 13.2 (2.0), 13.2 (1.9), 12.4 (1.6).LDPM: Perfusion (arbitrary units): Chest: 110.4 (32.1), 102.7 (27.8), 100.5 (28.1). Hand: 58.5 (26.2), 55.1 (17.5), 46.6 (14.9).DRS: Microvascular oxygen saturation (\%): Chest: 71.9 (7.3), 73.5 (12.2), 74.5 (9.2). Hand: 68.3 (11.5), 63.1 (11.6), 65.5 (11.7).

Conclusions: We were able to obtain noninvasive reproducible data for skin microvascular morphology, perfusion and oxygen saturation. The number of skin vessels is fewer in chest than in hand, with a slight reduction from day 1 to day 3 . There is higher perfusion in the chest than in the hand, probably due to higher skin temperature $\left(+2.8^{\circ}\right)$. Since all the infants has arterial oxygenation $>97 \%$, oxygen extraction in the skin microvessels were approximately $20 \%$ in the chest and $30 \%$ in the hand.

\section{IUGR AFFECTS LONG TERM POSTISCHEMIC NEOVASCULARIZATION CAPACITY}

F. Boubred ${ }^{1,2}$, B. Guillet ${ }^{2}$, I. Grandvuillemin', I. Ligi ${ }^{1}$, P. Pisano², F. Dignat-Georges ${ }^{2}$, U. Simeoni $i^{1,2}$

${ }^{1}$ Division of Neonatology, Hopital la Conception, AP-HM, ${ }^{2}$ INSERM UMR 608, Faculté de

Pharmacie, Université de la Méditerranée, Marseille, France

Introduction: Cardiovascular disease is a major cause of premature death. The severity of such diseases has been suggested to result from insufficient angiogenesis processes. Low birth weight is now recognized as a risk factor of mortality for such disease. However, the vascular repair capacity after ischemia in IUGR adult offspring is unknown.

Objective: To show how postischemic neovascularization capacity is affected in 12 mo-old adult IUGR rat offspring (maternal gestational low protein diet).

Material and methods: The unilateral hind limb ischemia model by femoral artery resection was used to study the neovascular capacity; the controlateral hind limb served as a reference for each animal. Laser Doppler imaging was used to assess blood flow perfusion in both hind limb after ischemia. Capillary densities of the anterior tibial muscle from both controlateral and ischemic limbs was measured at day 21 .

Results: After ischemia, IUGR offspring showed marked decreased blood flow in ischemic hind limb from day 1 (-60\% vs $-40 \%$ in IUGR and control offspring, $p<0.05)$. At day 21 , while ischemia promoted neovascularization in control offspring (+ $22 \%)$, vascular density was decreased in IUGR ischemic hind limb by $30 \%(p<0.05)$. Moreover, muscle capillary density of non-ischemic hind limb was higher in IUGR as compared with control offspring $(+20 \%, p<0.05)$.Conclusion: Postischemic neovascularization capacity following hind limb ischemia is affected in IUGR adult rat offspring. Angiogenic repair pathways, more than basal capillary density, may be affected in such condition. 